\title{
Do Performance Parameters Compare Between an Anaerobic Set and the 100-M Event in Swimming? ${ }^{+}$
}

\author{
Elissavet Terzi *, Ariadni Skari, Stefanos Nikolaidis, Konstantinos Papadimitriou, \\ Athanasios Kabasakalis and Vassilis Mougios \\ Laboratory of Evaluation of Human Biological Performance, School of Physical Education and Sport Science \\ at Thessaloniki, Aristotle University of Thessaloniki, 54124 Thessaloniki, Greece \\ * Correspondence: elizaterzi@hotmail.gr \\ + Presented at the 9th Greek Conference of Biochemistry and Physiology of Exercise, Thessaloniki, Greece, \\ 18-20 October 2019.
}

Published: 10 September 2019

\begin{abstract}
AIM: Anaerobic interval sets are commonly used in the training program of swimmers competing in short-distance events. However, data regarding the way that these sets compare to the competitive events are lacking. The aim of this study was to examine if there are differences in speed, blood lactate, stroke rate (SR), and stroke index (SI) between an anaerobic set of $4 \times 50 \mathrm{~m}$ maximal swimming with work-to-rest ratio of approximately 1:4 and the 100-m event in freestyle stroke. MATERIAL \& METHOD: Twenty-seven competitive swimmers (11 males, 16 females), aged $16.1 \pm 1.1$ years, completed the two tests on different days, in a random counterbalanced order. In each test, blood lactate was measured before and repeatedly after exercise through a portable lactate analyzer. Time and SR were recorded for each $50 \mathrm{~m}$ of the tests, and speed and SI were subsequently calculated. Three-way analysis of variance (time $\times$ test $\times$ gender) and Pearson's correlation analysis were used. The level of statistical significance was set at $\alpha=0.05$. RESULTS: Average speed was higher at $4 \times 50 \mathrm{~m}$ compared to $100 \mathrm{~m}(1.62 \pm 0.10$ and $1.56 \pm 0.10$, respectively, $p<0.001)$ and was correlated between tests $(r=0.930, p<0.001)$. Peak blood lactate after $4 \times 50 \mathrm{~m}$ was higher compared to $100 \mathrm{~m}(14.8 \pm 3.1$ and $10.9 \pm 3.3 \mathrm{mmol} / \mathrm{L}$, respectively, $p<0.001)$ and was correlated between tests $(r=0.640, p<0.001)$. Average SR was higher in $4 \times 50 \mathrm{~m}$ compared to $100 \mathrm{~m}$ $(47.0 \pm 3.6$ and $44.5 \pm 3.2$ cycles $/ \mathrm{min}$, respectively, $p<0.001)$ and was correlated between tests $(r=$ $0.836, p<0.001)$. Average SI did not differ but was correlated between tests $(r=0.937, p<0.001)$. Males had higher SR and SI and were faster than females $(p<0.05)$ but did not differ from females regarding lactate. CONCLUSIONS: Based on the aforementioned differences and correlations, the $4 \times 50 \mathrm{~m}$ training set could be used to improve the parameters that have impact on performance in the 100 -m event.
\end{abstract}

Keywords: anaerobic exercise; blood lactate; stroke index; stroke rate; swimming

(C) 2019 by the authors. Licensee MDPI, Basel, Switzerland. This article is an open access article distributed under the terms and conditions of the Creative Commons Attribution (CC BY) license (http://creativecommons.org/licenses/by/4.0/). 